\title{
0 efeito da aplicação de ligaduras funcionais no padrão de marcha e controlo postural em crianças hemiplégicas espásticas por paralisia cerebral
}

\author{
Angélica Almeida \\ Pedro Gonçalves \\ Maria Adília Silva \\ Leandro Machado
}

\author{
Faculdade de D esporto \\ Universidade do Porto \\ Portugal
}

\section{RESUMO}

Este trabalho centra-se na análise da marcha em crianças hemiplégicas espásticas por paralisia cerebral.

$\mathrm{Na}$ hemiplegia existem anomalias no padrão de marcha e no controlo postural. O uso de ligaduras funcionais (tapes) contribui como um meio de controlo do pé, para melhoria do padrão de marcha e da estabilidade postural.

Os objectivos principais deste estudo prendem-se com a quantificação dos efeitos na marcha, em crianças hemiplégicas, da colocação de ligaduras funcionais (forças de reacção ao solo, actividade muscular e parâmetros cinemáticos) e na estabilidade postural, imediatamente após a primeira colocação dos tapes e decorridos três meses de uso continuado.

Recorreu-se a uma amostra experimental composta por 7 crianças hemiplégicas de ambos os sexos e a um grupo controlo sem qualquer patologia. Para a análise da marcha utilizamos um sistema de vídeo $2 \mathrm{D}$, plataforma de forças e aparelho de electromiografia. $\mathrm{O}$ estudo cingiu-se à análise da fase de apoio do ciclo de marcha e do membro inferior afectado.

As principais conclusões retiradas deste estudo foram que a aplicação de ligaduras funcionais conduz a alterações no padrão de marcha e no controlo postural, promovendo um aumento da flexão dorsal do pé na fase de ataque ao solo e aumento da extensão do joelho na fase final de apoio, facilitando um aumento da actividade do músculo tibial-anterior e inibição parcial do tricípete sural na fase de ataque ao solo. Em suma, admitimos que os resultados obtidos após três meses de uso contínuo das ligaduras funcionais significaram alterações potencialmente positivas no padrão de marcha destas crianças.

\section{ABSTRACT \\ The ankle taping effects in gait and postural control in hemi- plegic spastic children with cerebral palsy}

This work addresses the gait analysis in spastic hemiplegic children with cerebral palsy.

In hemiplegia there are abnormalities in gait pattern and in postural control. Taping techniques are described as a way to control the foot and ankle, in order to improve the gait pattern and postural stability. The main purpose of this study is to quantify the ankle taping effects in gait ( ground reaction forces, muscle activity and kinematic parameters) and in postural stability immediately after the ankle taping application and after three months of continuous use, in hemiplegic children. The experimental sample was composed by 7 hemiplegic children, boys and girls, and the control group by 7 children without any pathology. For the gait analysis we used a 2D video system, a force platform and an electromyography device. This study dealt only with the analysis from the stance phase of the gait cycle of the affected inferior limb. The major conclusions arising from this study were that the ankle taping application produces changes on gait pattern and on postural control, icreasing the dorsiflexor movement during the final stance phase as well the knee extension at ground attack phase, facilitating an increase on the muscle tibial-anterior activity and parcial inibition of the tricipes sural during ground attack phase.

In summary, we admit that the attained results after three months of ankle taping continuous use denoted positive changes in these children gait pattern.

Key-Words: cerebral palsy, hemiplegia, ankle taping, gait pattern, postural control. 


\section{INTRODUÇÃO}

Este trabalho centra-se na análise da marcha em crianças com Paralisia Cerebral, remetendo-se especificamente para o estudo da hemiplegia. Tem como propósito analisar os efeitos da aplicação das ligaduras funcionais ${ }^{1}$ no pé e articulação tíbio-társica do membro inferior afectado, em crianças hemiplégicas, ao nível do padrão de marcha realizado e em termos de controlo postural.

A marcha humana é uma actividade que envolve, directa ou indirectamente, todo o corpo. Dado ser o segmento que faz o primeiro contacto com o solo, o pé reveste-se de uma importância particular. Tendo em conta a sua constituição anatómica e funcional, o pé é um complexo segmento do aparelho locomotor. Entre as suas funções destaca-se a capacidade de suportar, amortizar e distribuir a força, nas inúmeras situações em que é solicitado funcionalmente (16). A sensibilidade da superfície plantar do pé e respectivo feedback sensorial, desempenham importante função na selecção de uma resposta dinâmica do mesmo a alterações específicas provocadas pela aplicação de um determinado material, quer sejam sapatos, palmilhas ou tapes (22).

Neste trabalho tentaremos perceber o que sucederá na marcha em crianças com patologias do foro neurológico, nomeadamente com Paralisia cerebral, após utilização continuada de tapes.

Bartlett et al. (3) consideram a paralisia cerebral como um défice neuromuscular provocado por uma lesão não progressiva localizada numa ou em várias áreas do cérebro imaturo, resultando em alterações na função motora e na integridade sensorial. O quadro de paralisia cerebral pode apresentar-se sob várias formas, que em termos de topografia corporal poderão ser denominados de hemiplegia, diplegia ou tetraplegia, com variância em termos de qualidade de tónus (hipotonia / hipertonia ou espasticidade). A hemiplegia por paralisia cerebral, é o síndrome de paralisia cerebral mais comum entre crianças de termo e o segundo, depois da diplegia, entre as crianças de pré-termo (14). As crianças hemiplégicas, adquirem marcha na sua grande maioria, embora mais tardiamente que as crianças ditas normais (21). Para Bobath (4), a criança usa apenas o que sente e experiencia e se tais vivências sensório-motoras são anormais, a criança irá adaptar os padrões anormais de movimento para conseguir alguma funcionalidade, passando deste modo a perpetuá-los, reforçando-os através da repetição, que com o tempo conduzirá à instalação de contracturas e deformidades estruturais. A presença de espasticidade contribuí para a ausência de experiências sensoriais normais. Sabe-se que a espasticidade, oriunda de uma lesão ao nível do tracto cortico-espinal, é um factor limitador do movimento normal, trazendo repercussões em termos posturais e de marcha, na medida em que é responsável pela ausência de um estado de equilíbrio da actividade muscular entre os músculos agonista e antagonista que envolvem as articulações, bem como pela perda de um controlo muscular selectivo ${ }^{(23)}$. A espasticidade existente ao nível dos músculos da perna, origina uma postura de equino-varus do pé, que conduz a distúrbios nos padrões de movimento realizados ao nível das articulações do pé, tíbio-társica e joelho (25). A ausência de um controlo motor selectivo, responsável pela falta de equilíbrio na actividade muscular entre agonistas e antagonistas, influencia negativamente o correcto desenrolar do pé na fase de apoio (11).

No sentido de prevenir deformidades, promover uma postura mais correcta ao nível do pé, facilitando a execução de um padrão de marcha mais correcto e funcional, vários são os métodos referidos na bibliografia, e efectivamente utilizados, que são aplicados ao nível do pé e da articulação tíbio-társica, nomeadamente a aplicação de palmilhas ou colocação de tapes.

Cingindo-nos à análise de aplicação dos tapes, estes são frequentemente utilizados na actividade desportiva, tendo em conta os seus efeitos mecânicos pela contenção imposta à articulação, o efeito exteroceptivo pela estimulação de sensores cutâneos, o efeito psicológico e o efeito proprioceptivo fundamentado na eventual estimulação dos receptores musculares, tendinosos e capsulares, que por sua vez desencadeiam estímulos aferentes para o Sistema Nervoso Central (15).

Há indicações prementes quanto à utilização de tapes no pé equino-varo ou boto para correcção postural, num tratamento dito conservador (12).

Perry et al. (26), num estudo que tinha por objectivo analisar o efeito da aplicação dos tapes na superfície plantar, na estabilidade postural de uma população idosa, verificaram que a facilitação induzida pelos 
tapes conduz a um aumento da informação aferente a nível do Sistema Nervoso Central, acerca dos limites de estabilidade dentro da base de sustentação, isto numa posição ortoestática.

Em consonância, Perez et al. (25), num estudo efectuado com crianças diplégicas que realizavam marcha e tendo procedido à análise da actividade muscular do tibial-anterior e tricípete sural, através da electromiografia, servindo-se como factor a variar a colocação de tapes num único membro-inferior (pé e tíbio-társica), constataram uma diminuição do nível de espasticidade no membro sujeito a tape.

Com base no exposto, torna-se lícito verificar quais os efeitos da aplicação dos tapes num pé equinovarus, em crianças com um quadro motor de hemiplegia por Paralisia cerebral, quer ao nível do padrão de marcha realizado, quer em termos de controlo postural, procedendo para tanto a um estudo cinético, cinemático, electromiográfico e estabilométrico. Neste estudo cingimo-nos apenas à análise da fase de apoio do ciclo de marcha.

\section{MATERIAL E MÉTODOS}

\section{Amostra}

Recorreu-se a uma amostra experimental não aleatória por conveniência composta por 7 crianças hemiplégicas de ambos os sexos $(6.71 \pm 1.25$ anos, $21.14 \pm 5.37 \mathrm{~kg}$ e $1.15 \pm 0.06 \mathrm{~m})$ e a um grupo controlo composto por 7 crianças sem qualquer patologia, de ambos os sexos $(6.43 \pm 1.40$ anos, $23.61 \pm 3.93 \mathrm{~kg}$ e $1.18 \pm 0.05 \mathrm{~m})$.

\section{Instrumentos}

Para a análise da marcha utilizou-se uma câmara de vídeo, uma plataforma de forças (BERTEC tipo 4060-15) e um aparelho de electromiografia.

\section{Metodologia}

Para análise dos ciclos de marcha realizados por cada criança, optou-se pela execução de procedimentos de cariz cinemático, com recolha de imagens a 2D, no plano sagital; de cariz cinético, com o registo das Forças de Reacção do Solo; bem como pela utilização da electromiografia de superfície. Para a calibração do sistema de vídeo, recorreu-se à focagem manual do espaço onde decorreu o movimento, estando este preenchido por um dispositivo de calibração (gaiola metálica). Os três sistemas estavam sincronizados de modo a dispararem ao mesmo tempo, no início de cada evento (contacto do pé na plataforma). Os registos das forças (vertical, horizontal e médiolateral), foram obtidos através de uma plataforma de forças BERTEC 4060-15 (com 0.60m de comprimento e $0.40 \mathrm{~m}$ de largura), ligada a um amplificador BERTEC AM 6300, com ganhos pré-definidos e com uma frequência de amostragem de $1000 \mathrm{~Hz}$. O amplificador encontrava-se ligado à unidade de interface UM 100, que por sua vez conectava com o conversor analógico- digital de 16 bits (marca Biopac).

Os parâmetros cinemáticos foram quantificados através do A riel Performance A nalysis System. Os sinais obtidos pela plataforma de forças e pela electromiografia, foram processados através do programa Acqknowledge. Utilizou-se uma frequência de recolha de $50 \mathrm{~Hz}$ para o sistema de vídeo.

Para o registo da actividade muscular dos músculos agonista e antagonista, dos movimentos de flexão dorsal/flexão plantar do pé, foi utilizada a electromiografia de superfície.

Antecedendo a colocação dos eléctrodos, determinados requisitos ditados por Correia et al. (8) foram seguidos, no sentido de se reduzir a impedância entre pele/eléctrodo, contribuindo deste modo para uma recolha mais limpa do sinal. A salientar a limpeza da pele com álcool, no ponto de colocação do eléctrodo; aguardar um intervalo de tempo entre a colocação dos eléctrodos e o início da recolha, não inferior a 5 minutos.

Os eléctrodos de superfície activos, foram colocados sobre os músculos tibial anterior e tricípete sural, orientados paralelamente à orientação das fibras musculares, aconselhado por Correia et al. (8) para contracções isométricas, tendo o ponto de colocação correspondido ao ventre muscular de cada músculo. O eléctrodo terra, foi colocado numa superfície óssea, que neste caso correspondeu à rótula, do membro em análise (24).

Realizaram-se previamente alguns ensaios, de modo a que as crianças se sentissem mais familiarizadas com a instrumentação e com o próprio espaço. Foi solicitado a cada criança que caminhasse o mais naturalmente possível e que tentasse colocar apenas o pé hemiplégico na plataforma. A velocidade não foi controlada de modo a não provocar qualquer cons- 
trangimento no padrão de marcha realizado. Ao comando verbal "agora”, a criança deveria começar a caminhar. Foram realizadas múltiplas repetições até perfazer 6 repetições acertadas, completando um total de 10 segundos em cada repetição. Para a recolha do sinal estabilométrico, foi pedido a cada criança que se mantivesse imóvel durante 30 segundos, em cima da plataforma, com os pés juntos, braços ao longo do corpo e olhar em frente (protocolo utilizado por Duarte et al. (13); Barros et al. (2).

A parte prática comportou dois momentos distintos de recolha, sendo que no $1^{\circ}$ momento procedeu-se à avaliação do padrão de marcha e do controlo postural sem tapes e após a primeira aplicação dos mesmos. No $2^{\circ}$ momento, passados 3 meses de uso sistemático dos tapes por parte das crianças pertencentes ao grupo experimental, efectuou-se nova recolha com a utilização dos mesmos. Realizou-se ainda a análise dos padrões de marcha e do controlo postural referente ao grupo controlo. No grupo controlo, optou-se pela análise do membro inferior direito por conveniência da orientação dos eixos da plataforma de forças e pela colocação da câmara de vídeo. De referir que o estudo cingiu-se apenas à análise da fase de apoio do ciclo de marcha e do membro inferior afectado.

Durante três meses, procedeu-se à aplicação de uma técnica de tape, inibitória da postura patológica de equino-varus a nível do pé e respectiva articulação tíbio-társica. Esta técnica foi aplicada uma vez por semana, sendo que o tape poderá manter-se durante 3 dias sem ser removido, mantendo as suas propriedades correctivas, de tracção e de informação proprioceptiva (17). A sua aplicação teve como função a correcção e manutenção do pé, sendo que através das forças de tracção impostas pelos tapes, o pé era levado para uma posição de eversão e dorsi-flexão, promovendo-se um alongamento do tendão de aquilles. Foram colocadas tiras de suporte na parte distal da perna a nível dos metatarsos; promoveu-se uma correcção do calcâneo para eversão com a colocação de uma tira longitudinal; correcção do arco plantar interno, controlando a inversão do pé, sendo a direcção do tape de fora para dentro; aplicação de uma tira na face dorso-externa do pé de modo a estimular o músculo curto-extensor dos dedos, inibindo a flexão dos dedos; reforço dos apoios colocados anteriormente, com estabilização da articulação tíbio-társica.

\section{Processamento dos dados}

Para o processamento das curvas de força Fy (antero-posterior) e $\mathrm{Fz}$ (vertical), procedeu-se à sua filtragem utilizando um filtro de passa baixo a $40 \mathrm{~Hz}$, janela de Hamming, tendo sido tirado o offset de cada curva, e normalização pelo peso de cada criança. Os sinais electromiográficos referentes aos músculos tibial-anterior e tricípete sural foram filtrados aplicando-se um filtro passa banda de $25 \mathrm{~Hz}$ a $500 \mathrm{~Hz}$, janela $\mathrm{H}$ amming ${ }^{(8)}$. Procedeu-se de seguida à rectificação das curvas, através da função ABS e aplicou-se o Envelope Linear com um filtro passa baixo de $6 \mathrm{~Hz}$ (30). As curvas foram ainda normalizadas ao valor do pico máximo apresentado por cada curva, tal como sugerido por Correia et al. (8).

Para análise dos registos cinéticos e electromiográficos obtidos durante a fase de apoio, procedeu-se à divisão da referida fase, tendo por base Fy (Força antero-posterior), em sub-fases distintas (1, 2, 3, 4, A, B), como se pode observar na Figura 1 .

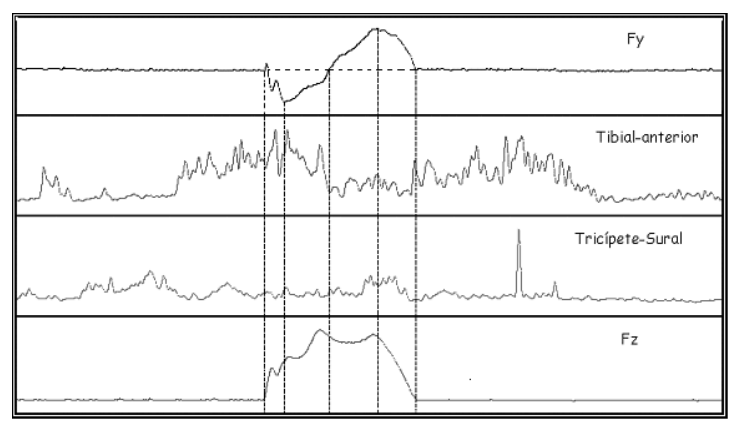

Figura 1. Delimitação das sub-fases dentro da fase de apoio nas curvas de força (Fy e Fz) e nos traçados electromiográficos.

As fases foram delimitadas segundo Fy, do seguinte modo:

Fase 1 - fase de ataque ao solo (desde o ponto zero até ao valor mínimo encontrado por análise de Fy, verificando-se o início de transferência de carga sobre o membro inferior por observação de Fz); Fase 2 - fase média inicial de apoio (desde o mínimo encontrado até ao valor zero segundo Fy, observando-se uma aceitação da carga sobre o membro, segundo $\mathrm{Fz}$ );

Fase 3 - fase média final de apoio (desde o valor zero até ao valor máximo encontrado, verificando-se 
a passagem da fase média de apoio para o início da fase de push off, segundo $\mathrm{Fz}$ );

Fase 4 - fase final de apoio (desde o valor máximo até ao valor zero para Fy, correspondendo à fase do push off do pé em relação ao solo, segundo Fz);

Fase $\mathrm{A}$ - fase de travagem (corresponde à união das fases 1 e 2 );

Fase B - fase de aceleração (junção das fases 3 e 4).

\section{Metodologia Estatística}

Aplicaram-se testes não paramétricos, sendo que, as variáveis na sua maioria, não demonstraram uma distribuição normal, como foi verificado através do teste de Kolmogorov Smirnov.

Deste modo, utilizou-se o teste de Wilcoxon para amostras dependentes ( Amostra Experimental ( $1^{\mathrm{a}}$ Recolha sem tape Vs $1^{\text {a }}$ recolha com tape; $1^{\text {a Recolha }}$

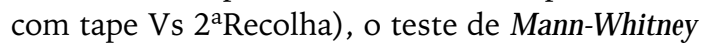
para amostras independentes (Grupo Controlo Vs Amostra Experimental ( $1^{\mathrm{a}}$ recolha sem tape/ $2^{\mathrm{a}}$ recolha), para um nível de significância $\alpha=0,05$. De referir, que se procedeu ainda à determinação do Coeficiente de Correlação (R) para análise de possível relacionamento entre algumas variáveis dentro da mesma recolha, com um nível de significância de $\alpha=0,05$.

\section{RESULTADOS}

A análise das diferentes recolhas, mostrou diferenças estatisticamente significativas nas variáveis estudadas, quer para a fase de apoio do ciclo de marcha, quer para a migração do centro de pressão, referente ao estudo do controlo postural, para um valor de $\alpha=0,05$.

\section{Apresentação dos Resultados}

Os resultados obtidos nas diferentes recolhas realizadas, para as diversas variáveis estudadas - cinéticas, cinemáticas e electromiográficas - são apresentados nos Quadros que se seguem. A respectiva análise estatística será feita na secção seguinte.

Quadro 1. Estatística descritiva - média \pm desvio padrão - relativo às variáveis cinéticas correspondentes às forças antero-posterior (IntFy) e vertical (IntFz e MáxFz] em todas as sub-fases para a $1^{a}$ Recolha Sem Tape (ST) e Com Tape (CT), $2^{a}$ Recolha [ $2^{a} R$ ) e Grupo Controlo (C) (Int-fracção do Impulso Total; Máx-em unidades de peso corporal].

\begin{tabular}{lcccccc}
\hline Variáveis & Fase 1 & Fase 2 & Fase 3 & Fase 4 & Fase A & Fase B \\
\hline IntFy ST & $.0063 \pm .0012$ & $.0103 \pm .0057$ & $.0144 \pm .0068$ & $.0076 \pm .0025$ & $.0165 \pm .0064$ & $.0216 \pm .0083$ \\
IntFy CT & $.0054 \pm .0009$ & $.0100 \pm .0028$ & $.0159 \pm .0060$ & $.0091 \pm .0044$ & $.0154 \pm .0026$ & $.0235 \pm .0091$ \\
IntFy 2åR & $.0056 \pm .0019$ & $.0091 \pm .0034$ & $.0163 \pm .0041$ & $.0117 \pm .0055$ & $.0148 \pm .0051$ & $.0279 \pm .0088$ \\
IntFy C & $.0058 \pm .0033$ & $.0109 \pm .0077$ & $.0398 \pm .0433$ & $.0090 \pm .0032$ & $.0167 \pm .0096$ & $.0342 \pm .0319$ \\
\hline IntFz ST & $.0543 \pm .0167$ & $.2138 \pm .0609$ & $.2648 \pm .0839$ & $.0924 \pm .0295$ & $.2680 \pm .0540$ & $.3607 \pm .0807$ \\
IntFz CT & $.0531 \pm .0310$ & $.2017 \pm .0590$ & $.2775 \pm .0517$ & $.0899 \pm .0337$ & $.2528 \pm .0636$ & $.3606 \pm .0865$ \\
IntFz 2ä & $.0493 \pm .0121$ & $.1822 \pm .0526$ & $.2686 \pm .0426$ & $.0963 \pm .0346$ & $.2315 \pm .0519$ & $.3648 \pm .0459$ \\
IntFz C & $.0872 \pm .0544$ & $.2781 \pm .1540$ & $.3305 \pm .1558$ & $.0635 \pm .0151$ & $.3653 \pm .1656$ & $.3938 \pm .1628$ \\
\hline MáxFz ST & $.6948 \pm .0738$ & $1.022 \pm .0767$ & $1.034 \pm .0849$ & $.8764 \pm .0876$ & $1.024 \pm .0750$ & $1.034 \pm .0849$ \\
MáxFz CT & $.7113 \pm .1123$ & $1.022 \pm .0791$ & $1.040 \pm .0915$ & $.8569 \pm .0815$ & $1.022 \pm .0793$ & $1.021 \pm .1294$ \\
MáxFz 2äR & $.6980 \pm .1340$ & $1.010 \pm .0902$ & $1.059 \pm .0872$ & $.9035 \pm .1213$ & $1.011 \pm .0902$ & $1.064 \pm .0956$ \\
MáxFz C & $.7099 \pm .1361$ & $.9816 \pm .0741$ & $.9982 \pm .0487$ & $.7906 \pm .0239$ & $.9870 \pm .0737$ & $.9998 \pm .0468$ \\
\hline
\end{tabular}


Quadro 2. Estatística descritiva - média \pm desvio padrão - relativo às variáveis cinemáticas representadas pela média das amplitudes articulares em graus ( ${ }^{\circ}$ ] obtidas na articulação do joelho (MédAjoelho] e tíbio-társica (MédAtornz) em todas as sub-fases para a $1^{a}$ Recolha Sem (ST) e Com Tape (CT), $2^{a}$ Recolha ( $2^{a} R$ ) e Grupo Controlo (C).

\begin{tabular}{lcccccc}
\hline Variáveis & Fase 1 & Fase 2 & Fase 3 & Fase 4 & Fase A & Fase B \\
\hline MédAjoelho ST & $164.50 \pm 10.02$ & $164.56 \pm 8.87$ & $168.60 \pm 6.89$ & $153.73 \pm 10.33$ & $164.55 \pm 9.19$ & $161.47 \pm 7.60$ \\
MédAjoelho CT & $164.52 \pm 8.96$ & $165.70 \pm 8.94$ & $170.04 \pm 6.52$ & $156.34 \pm 10.05$ & $165.51 \pm 8.69$ & $163.82 \pm 7.37$ \\
MédAjoelho 2ㅁ & $161.93 \pm 4.24$ & $159.89 \pm 4.69$ & $166.05 \pm 5.14$ & $156.87 \pm 5.61$ & $160.77 \pm 3.48$ & $161.98 \pm 4.61$ \\
MédAjoelho C & $168.73 \pm 4.75$ & $163.49 \pm 6.84$ & $160.25 \pm 7.83$ & $144.79 \pm 7.03$ & $165.84 \pm 5.37$ & $165.84 \pm 5.36$ \\
\hline MédAtornz ST & $127.21 \pm 6.23$ & $121.19 \pm 7.78$ & $114.84 \pm 7.86$ & $115.25 \pm 5.74$ & $123.60 \pm 7.26$ & $114.90 \pm 6.79$ \\
MédAtornz CT & $125.31 \pm 5.15$ & $120.13 \pm 7.05$ & $113.44 \pm 6.31$ & $114.66 \pm 4.33$ & $122.41 \pm 6.08$ & $113.88 \pm 5.26$ \\
MédAtornz 2åR & $119.11 \pm 4.43$ & $113.90 \pm 3.84$ & $107.71 \pm 6.11$ & $109.89 \pm 7.24$ & $116.08 \pm 3.73$ & $108.53 \pm 6.31$ \\
MédAtornz C & $117.79 \pm 2.72$ & $112.89 \pm 6.54$ & $102.78 \pm 7.89$ & $108.66 \pm 6.86$ & $115.39 \pm 3.74$ & $115.39 \pm 3.74$ \\
\hline
\end{tabular}

Quadro 3. Estatística descritiva - média \pm desvio padrão - relativo às variáveis Electromiográficas representadas pelo valor do Integral normalizado ao pico máximo, fracção do Impulso Total [Int], IntTA (Integral do tibial anterior], IntTS (Integral do tricípete sural) obtidas na $1^{a}$ Recolha Sem Tape [ST] e Com Tape [CT), $2^{a}$ Recolha $\left(2^{a} R\right)$ e Grupo Controlo $(C)$.

\begin{tabular}{lcccccc}
\hline Variáveis & Fase 1 & Fase 2 & Fase 3 & Fase 4 & Fase A & Fase B \\
\hline IntTAST & $.0743 \pm .0289$ & $.0919 \pm .0170$ & $.0920 \pm .0474$ & $.0917 \pm .0369$ & $.1665 \pm .0280$ & $.1854 \pm .0794$ \\
IntTACT & $.0699 \pm .0281$ & $.0893 \pm .0373$ & $.0906 \pm .0389$ & $.0804 \pm .0298$ & $.1594 \pm .0855$ & $.1710 \pm .0982$ \\
IntTA2 2aR & $.0694 \pm .0198$ & $.0729 \pm .0277$ & $.0847 \pm .0460$ & $.0670 \pm .0287$ & $.1425 \pm .0368$ & $.1549 \pm .0633$ \\
IntTAC & $.0914 \pm .0262$ & $.1038 \pm .0372$ & $.0935 \pm .0623$ & $.0441 \pm .0200$ & $.1960 \pm .0261$ & $.1376 \pm .0652$ \\
\hline IntTS ST & $.0647 \pm .0173$ & $.0962 \pm .0323$ & $.1250 \pm .0509$ & $.0870 \pm .0376$ & $.1611 \pm .0290$ & $.2126 \pm .0440$ \\
IntTS CT & $.0578 \pm .0256$ & $.0873 \pm .0345$ & $.1399 \pm .0324$ & $.0869 \pm .0358$ & $.1439 \pm .0683$ & $.2232 \pm .0748$ \\
IntTS 2aR & $.0587 \pm .0214$ & $.0801 \pm .0285$ & $.1364 \pm .0418$ & $.0792 \pm .0223$ & $.1343 \pm .0418$ & $.2137 \pm .0538$ \\
IntTSC & $.0683 \pm .0236$ & $.1215 \pm .0706$ & $.1666 \pm .0768$ & $.0478 \pm .0182$ & $.1900 \pm .0743$ & $.2146 \pm .0819$ \\
\hline
\end{tabular}

Quadro 4. Estatística descritiva - média ( $m$ ?) \pm desvio padrão - relativo aos valores da área de migração do Centro de Pressão definido por crianças nas diferentes recolhas: para o grupo Experimental, na $1^{a}$ recolha Sem Tape (E1 ${ }^{a} R$ $S T$ ] e Com Tape $\left[\varepsilon 1^{a} R C T\right]$ e na $2^{a}$ recolha $\left(\varepsilon 2^{a} R\right]$ e para o Grupo Controlo.

\begin{tabular}{lc}
\hline Recolhas & Média \pm Desvio Padrão \\
\hline EläR ST & $.0013 \pm .0006$ \\
EläR CT & $.0012 \pm .0006$ \\
E2 ${ }^{a}$ R & $.0024 \pm .0015$ \\
Controlo & $.0009 \pm .0002$ \\
\hline
\end{tabular}

\section{Análise entre $1^{\mathrm{a}}$ recolha Sem Tape e Com Tape}

A descrição dos valores apresentados, remeter-se-á na sua maioria às variáveis em que se obtiveram diferenças significativas.

São evidentes diferenças entre a $1^{\text {a }}$ recolha sem e com tape na componente antero-posterior (Fy) das forças de reacção do solo apenas na fase de ataque ao solo (Fase 1) (Quadro 5).
Quadro 5. Apresentação dos valores de prova $(p)$ das variáveis cinéticas correspondentes às forças antero-posterior [ IntFy] e vertical $[\operatorname{lntFz}$ ] e das variáveis cinemáticas representadas pela média das amplitudes articulares obtidas na articulação do joelho [MédAjoelho] e tíbio-társica [MédAtornz] em todas as sub-fases para a $1^{a}$ Recolha Sem (ST) e Com Tape (CT)

Teste de Wilcoxon (p)

Variáveis Fase 1 Fase 2 Fase 3 Fase 4 Fase A Fase B

\begin{tabular}{lllllll}
\hline IntFy: & $0.018^{*}$ & 0.866 & 0.310 & 0.310 & 0.799 & 0.499
\end{tabular}

ST Vs CT

$\begin{array}{lllllll}\text { IntFz: } & 0.866 & 0.866 & 0.612 & 0.499 & 0.499 & 1.000\end{array}$

ST vs CT

$\begin{array}{llllll}\text { MédAjoelho: } \quad 0.866 & 0.176 & 0.128 & 0.043^{*} & 0.398 & 0.018 *\end{array}$ ST vs CT

MédAtornz: $\quad 0.091 \quad 0.499 \quad 0.310 \quad 0.735 \quad 0.735 \quad 0.398$ ST vs CT

* valor estatisticamente significativo $p<\alpha=0,05$ 
Apenas na fase final de apoio se verificaram alterações na amplitude articular a nível do joelho, como se pode observar no Quadro 5, com aumento da amplitude média de extensão do mesmo, a verificar no Quadro 2.

\section{Análise entre $1^{\mathrm{a}}$ Recolha Com Tape e $2^{\mathrm{a}}$ Recolha}

Como verificamos no Quadro 6, as alterações foram notórias nomeadamente nos parâmetros cinemáticos avaliados, especificamente ao nível da articulação tíbio-társica, com aumento do movimento de dorsiflexão na fase de ataque ao solo (Fase1), tal como observado no Quadro 2. Foram visíveis diferenças na componente vertical de força $(\mathrm{Fz})$ na fase média final de apoio (Fase 3), como se pode observar no Quadro 6.

Quadro 6. Representação dos valores de prova ( $p$ ) das variáveis cinéticas correspondentes às forças antero-posterior (IntFy) e vertical (MáxFz) e das variáveis cinemáticas representadas pela média das amplitudes articulares obtidas na articulação do joelho (MédAjoelho], tíbio-társica [MédAtornz] e pé (MédApé] em todas as sub-fases para a $1^{a}$ Recolha Com Tape (CT) e $2^{a}$ Recolha $\left(2^{a} R\right.$ ].

\begin{tabular}{|c|c|c|c|c|c|c|}
\hline \multirow[b]{2}{*}{ Variás } & \multicolumn{4}{|c|}{ Teste de Wilcoxon (p) } & \multirow[b]{2}{*}{ Fase A } & \multirow[b]{2}{*}{ Fase B } \\
\hline & Fase 1 & Fase 2 & Fase 3 & Fase 4 & & \\
\hline $\begin{array}{l}\text { IntFy: } \\
\text { CT vs 2äR }\end{array}$ & 0.612 & 0.735 & 0.866 & 0.063 & 735 & $0.043 *$ \\
\hline $\begin{array}{l}\text { MáxFz: } \\
\text { CT vs 2ạR }\end{array}$ & 0.735 & 0.866 & $0.028 *$ & 0.091 & 0.866 & $0.028 *$ \\
\hline $\begin{array}{l}\text { MédAjoelho: } \\
\text { CT vs 2äR }\end{array}$ & 0.176 & 0.091 & 0.063 & 0.866 & 0.063 & 0.310 \\
\hline $\begin{array}{l}\text { MédAtornz: } \\
\text { CT vs 2ªR }\end{array}$ & $0.018 *$ & $0.018^{*}$ & $0.018 *$ & 0.237 & $0.018 *$ & $0.018 *$ \\
\hline
\end{tabular}

$*$ valor estatisticamente significativo $p<\alpha=0,05$

Constatou-se a presença do efeito de co-activação entre a actividade muscular exibida pelos músculos tibial-anterior e tricípete sural, comprovado pelo índice de correlação $\left(\mathrm{R}_{\left(2^{\mathrm{a} R e c}\right)}=0,769\right.$ e $\mathrm{R}_{\left(1^{\mathrm{a}} \mathrm{Rec}\right)}=$ 0,610 ), observado nas figuras 2 e 3 , verificando-se um aumento da actividade do tibial-anterior e inibição parcial do tricípete sural.

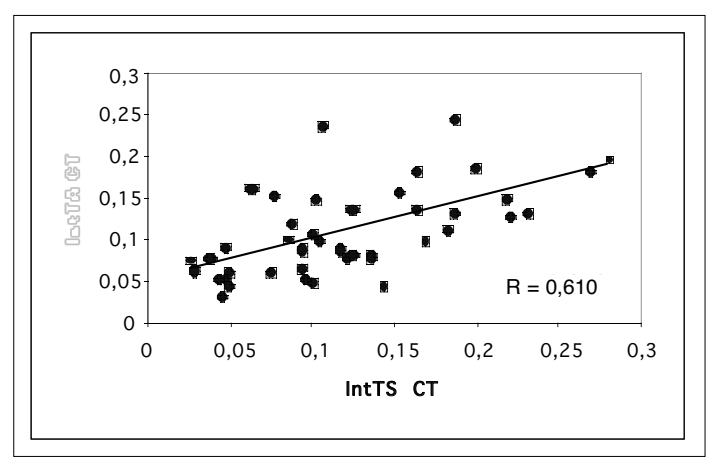

Figura 2. Gráfico de dispersão com os valores da variável Integral referente aos músculos Tibial-anterior (IntTA CT) e tricípete sural (IntTS CT) na $1^{a}$ Recolha Com Tape.

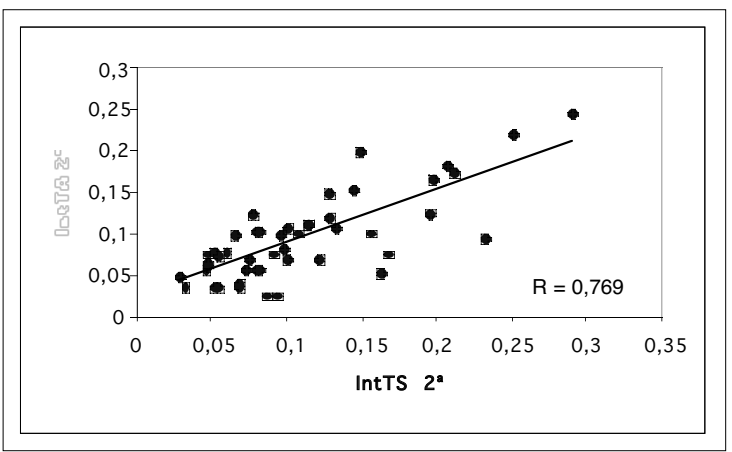

Figura 3. Gráfico de dispersão com os valores da variável Integral referente aos músculos Tibial-anterior (IntTA $2^{\circ}$ ) e tricípete sural (IntTS $2^{\circ}$ ) na $2^{a}$ Recolha.

Análise entre a $1^{\mathrm{a}}$ Recolha Sem Tape vs Grupo Controlo e $2^{a}$ Recolha vs Grupo Controlo

Verificam-se diferenças tanto em termos cinemáticos, como ao nível da actividade electromiográfica, referente à análise entre a $1^{\text {a }}$ Recolha Sem Tape e Grupo Controlo, como se pode observar no Quadro 7. 
Quadro ?. Representação dos valores de prova ( $p$ ) obtidos entre Grupo Controlo (C) Vs $1^{a}$ Recolha Sem Tape (ST) e Controlo Vs $2^{a}$ Recolha $\left(2^{a} R\right)$ para as variáveis cinéticas (IntFy - integral de Fy; MáxFy - valor máximo de Fy), cinemáticas (MédAjoelho - média de amplitude da articulação do joelho) e electromiográficas (IntTA - integral do tibial anterior; IntTS - integral do tricípete sural).

\begin{tabular}{|c|c|c|c|c|c|c|}
\hline Yarí, & Tes & de $M a$ & nn-Whitn & ey (p) & $\cos$ & $P$ \\
\hline $\begin{array}{l}\text { IntFy: } \\
\text { C Vs ST }\end{array}$ & 0.306 & 0.848 & 0.225 & 0.482 & 0.655 & 0.565 \\
\hline $\begin{array}{l}\text { IntFy: } \\
\text { C Vs 2-aR }\end{array}$ & 0.949 & 0.944 & 0.655 & 0.277 & 0.949 & 0.482 \\
\hline $\begin{array}{l}\text { MáxFy: } \\
\text { Cvs ST }\end{array}$ & 0.277 & 0.277 & 0.406 & 0.406 & 0.848 & 0.406 \\
\hline $\begin{array}{l}\text { MáxFy: } \\
\text { Cvs 2äR }\end{array}$ & 0.655 & 0.277 & 0.949 & 0.949 & 0.655 & 0.949 \\
\hline $\begin{array}{l}\text { MédAjoelho: } \\
\text { C vs ST }\end{array}$ & 0.655 & 0.749 & 0.064 & 0.110 & 0.848 & 0.225 \\
\hline $\begin{array}{l}\text { MédAjoelho: } \\
\text { Cvs 2aR }\end{array}$ & $0.013^{*}$ & 0.225 & $0.048 *$ & $0.009 *$ & 0.142 & $0.048^{x}$ \\
\hline $\begin{array}{l}\text { IntTA: } \\
\text { C vs ST }\end{array}$ & 0.406 & 0.749 & 0.949 & $0.025^{*}$ & $0.048^{*}$ & 0.180 \\
\hline $\begin{array}{l}\text { IntTA: } \\
\text { C vs 2 }{ }^{-a R}\end{array}$ & 0.110 & 0.180 & 0.949 & 0.110 & $0.025^{*}$ & 0.482 \\
\hline $\begin{array}{l}\text { IntTS: } \\
\text { C vs ST }\end{array}$ & 0.848 & 0.482 & 0.225 & $0.035^{*}$ & 0.482 & 0.655 \\
\hline $\begin{array}{l}\text { IntTS: } \\
\text { CVs 2 }{ }^{-a R}\end{array}$ & 0.337 & 0.225 & 0.406 & $0.025 *$ & 0.180 & 0.749 \\
\hline
\end{tabular}

Mesmo após 3 meses de uso dos tapes, as crianças do grupo experimental denotam algumas diferenças em relação às do Grupo Controlo. Porém denotam-se melhorias, comparativamente com a $1^{\text {a }}$ Recolha, nomeadamente ao nível dos parâmetros cinéticos e cinemáticos verificando-se uma aproximação dos valores apresentados na $2^{\mathrm{a}}$ Recolha aos do Grupo Controlo.

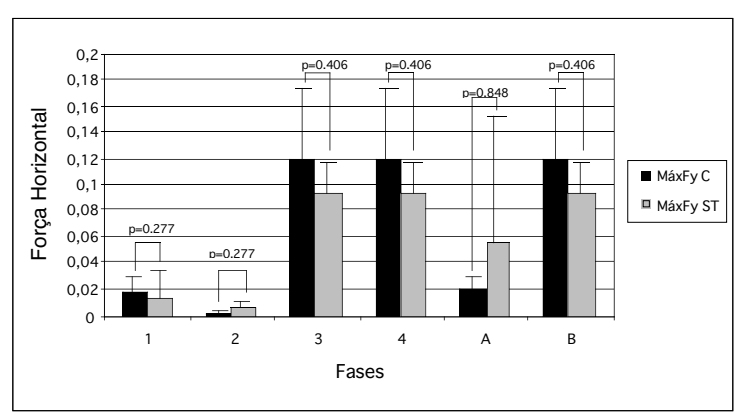

Figura 4. Comparação dos valores da força de reacção do solo na direcção antero - posterior entre o Grupo Controlo e a $1^{a}$ Recolha Sem Tape.

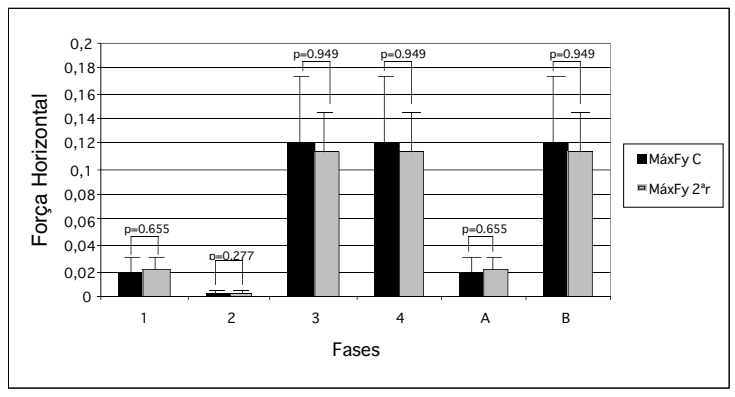

Figura 5. Representação gráfica da força de reacção do solo na direcção antero-posterior para o Grupo Controlo e $2^{a}$ Recolha.

No que se refere à variável MáxFy (Força antero-posterior) verificam-se melhorias da $1^{\text {a }}$ Recolha Sem Tape para a $2^{\text {a }}$ Recolha, evidenciadas pelos valores de prova (p) que são superiores aos apresentados na análise entre o Grupo Controlo e $1^{\text {a }}$ Recolha

(Figuras 4 e 5).

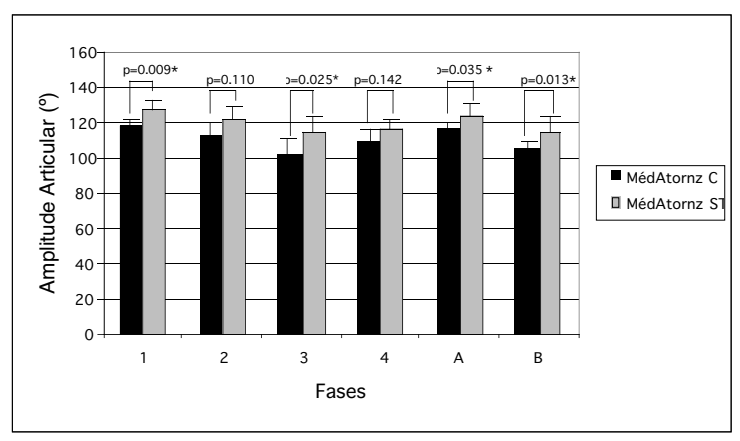

Figura 6. Comparação das amplitudes articulares obtidas para a articulação tíbio-társica entre o Grupo Controlo e $1^{a}$ Recolha Sem Tape.

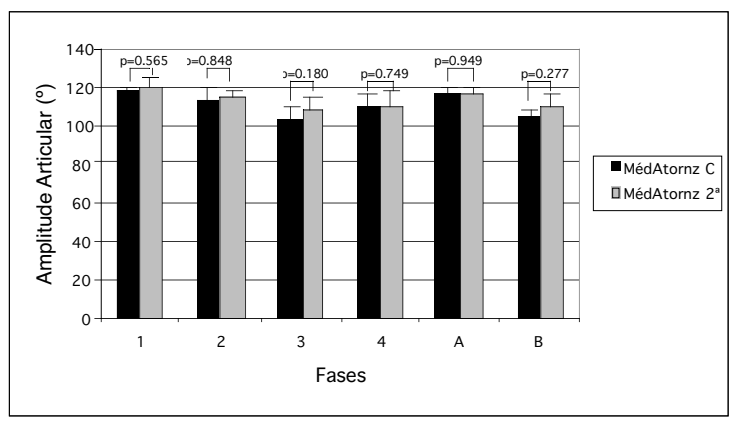

Figura ?. Comparação das amplitudes articulares obtidas para a articulação tíbio-társica entre o Grupo Controlo e $2^{a}$ Recolha. 
As melhorias são notórias em termos de amplitude média apresentada pela articulação tíbio-társica na $2^{\text {a }}$ Recolha, sendo que já não se evidenciam diferenças estatisticamente significativas entre o Grupo Controlo e a $2^{\text {a }}$ Recolha, ao contrário do que foi obtido na $1^{\text {a }}$ Recolha, como se pode observar nas Figuras 6 e 7 .

\section{Análise dos valores Estabilométricos}

Quanto à análise da migração do centro de pressão, verificaram-se alterações entre a $1^{\text {a }}$ Recolha com Tape e a $2^{\text {a }}$ Recolha, com aumento notório do valor médio da área de migração na $2^{\mathrm{a}}$ Recolha, e entre a $2^{\mathrm{a}}$ Recolha e o Grupo Controlo.

Quadro 8. Valores do testes estatísticos aplicados entre as diferentes recolhas para o Grupo Experimental $[\varepsilon]$ e Grupo Controlo.

\begin{tabular}{|c|c|}
\hline Recolhas & Teste Estatístico (p) \\
\hline E1 aRSTVs E1 aRCT & 0.753 \\
\hline E1 ${ }^{a}$ RCT Vs E2 ${ }^{a}$ R & $0.018 *$ \\
\hline Controlo Vs E1 aRST & 0.199 \\
\hline Controlo Vs E2 ${ }^{\mathrm{a}} \mathrm{R}$ & $0.013 *$ \\
\hline
\end{tabular}

* valor estatisticamente significativo $p<\alpha=0.05$

\section{DISCUSSÃO}

Sobre a análise efectuada entre a $1^{\text {a }}$ Recolha Sem Tape e Com Tape, no que se refere à análise cinética, sabendo que a componente antero-posterior da força de reacção do solo descreve a progressão do pé, a existência de diferenças a este nível, leva-nos a pensar que o movimento realizado com o pé para a frente na fase de ataque ao solo, foi mais controlado aquando do uso de tape e a força necessária para provocar uma desaceleração do movimento do pé foi menor. Estes resultados vão de acordo ao comentário de Cordova et al. (7), ao referirem que a estabilidade induzida pela aplicação dos tapes, permite que as forças impostas ao complexo pé tíbio-társica sejam atenuadas. Quanto à análise cinemática, os resultados sugerem um aumento da amplitude média de extensão do joelho na fase final de apoio, desde a primeira aplicação dos tapes. Estudos hão que ao referirem uma diminuição na ocorrência de uma flexão plantar prematura na fase de push-off, com a aplicação de ortóteses, salientam mudanças que reflectem uma transição mais suave do centro de pressão do médio-pé para o ante-pé, o que implica um aumento da amplitude de extensão do joelho ${ }^{(1,19)}$.

Relativamente à análise efectuada entre os resultados da $1^{\text {a }}$ Recolha Com Tape e da $2^{\text {a }}$ Recolha (passados 3 meses de uso contínuo dos tapes), constatouse um aumento da força média na fase 3 , ou fase média-final de apoio, na $2^{\text {a }}$ recolha, o que indica um aumento da transferência de carga para o membro inferior hemiplégico, encontrando-se de acordo com os resultados obtidos por Condie et al. (6), facto também constatado por Machado (18) ao verificar um aumento da transferência de peso na fase de aceleração com o uso de palmilhas proprioceptivas ao fim de 3 meses de uso sistemático. No que se refere à análise cinemática, verificou-se que o uso sistemático dos tapes conduziu a um aumento da amplitude média de dorsi-flexão da tíbio-társica na fase de ataque ao solo, o que vai de encontro aos resultados de Thomas et al. (29) ao salientarem melhorias na amplitude do movimento ocorrido ao nível do tornozelo (tíbio-társica), embora estes resultados tenham sido obtidos com a aplicação de ortóteses em crianças com a mesma patologia que as do presente estudo. Além do mais, poderemos supor que o aumento da amplitude de dorsi-flexão poderá também dever-se a uma diminuição da actividade muscular do tricípete sural. Sabe-se que quando ocorre uma contracção exagerada do mesmo, esta impede o desenrolar do movimento anterior da tíbia na fase de apoio (21). Considerando que um dos efeitos da aplicação dos tapes será o aumento da estimulação muscular, potenciando a sua função (20), então compreendemos que a aplicação dos tapes tenha conduzido a uma coactivação entre os músculos tibial-anterior e tricípete sural, que se reflecte num aumento da contracção muscular do tibial-anterior, havendo uma inibição parcial da actividade do tricípete sural. $\mathrm{Na}$ comparação dos valores entre a $2^{\mathrm{a}}$ Recolha e o Grupo Controlo, as diferenças não são já tão notórias como as constatadas aquando da $1^{\text {a }}$ Recolha. Assim sendo, apesar de se encontrarem diferenças na componente vertical da força de reacção do solo, resultado já obtido por Condie et al. (6) ao referirem uma diminuição das forças de impacto na fase inicial de apoio com a aplicação de ortóteses, no que concerne à análise cinemática, apenas foram visíveis 
diferenças ao nível das amplitudes articulares realizadas pela articulação do joelho. Apesar de Abel et al. (1) referirem um melhor alinhamento articular, e o controlo consequente induzido a nível distal poderá exercer um efeito positivo nas articulações proximais, o facto é que os efeitos deletérios provocados pelos músculos espásticos nas articulações proximais (neste caso o joelho), não são totalmente eliminados pelo uso deste sistema de contenção. $\mathrm{O}$ aumento da flexão observado a nível do joelho, poderá ter origem na espasticidade apresentada pelos músculos que rodeiam esta articulação, nomeadamente os ísquio-tibiais e o tricípete sural (9). Ao contrário do obtido entre $1^{\mathrm{a}}$ recolha e grupo controlo, nesta análise não se verificaram diferenças nas amplitudes médias articulares ao nível da tíbiotársica, o que evidencia uma diminuição na postura em equino ou flexão plantar, aproximando-se de amplitudes articulares ditas normais aqui representadas e referenciadas pelo grupo controlo.

No que concerne à análise dos valores estabilométricos obtidos, será de salientar que entre a $1^{\mathrm{a}}$ recolha com tape e a $2^{\text {a }}$ recolha, verificaram-se alterações significativas, ao ser notório um aumento do valor médio da área de migração do centro de pressão na $2^{\mathrm{a}}$ recolha. Celso et al. (5) sugerem uma explicação para este facto ao referirem que uma maior área de deslocamento poderá ser resultado de uma estratégia do sistema nervoso para receber mais informações espaciais para a manutenção do equilíbrio.

Reforçando esta ideia, Schieppati et al. (27) afirmam que uma área de deslocamento do centro de pressão maior poderá estar relacionada com um controle postural mais efectivo, quando associada a um limite de estabilidade maior. $\mathrm{Na} 2^{\mathrm{a}}$ recolha, verificaram-se valores médios da área de migração do centro de pressão superiores ao do grupo controlo, o que poderá significar neste caso prático, que houve uma necessidade por parte do Sistema Nervoso Central, em recolher o máximo de informações sensoriais para uma posterior aquisição e integração da competência motora, que neste caso se tratava em manter o equilíbrio, servindo-se do mecanismo de feedback. Sussman (28), refere que durante a fase de transição de um padrão de movimento considerado preferido, para outro desconhecido ou pouco praticado, o sistema encontra-se num estado de instabilidade, até se estabelecer o novo padrão de movimento. Tal facto leva-nos a supor que o período de tempo determinado para a aplicação sistemática dos tapes, poderá não ter sido suficiente para que um novo padrão de movimento se pudesse estabelecer, estando ainda dependente do mecanismo de feedback.

\section{CONCLUSÃO}

As principais conclusões retiradas deste estudo foram que a aplicação de ligaduras funcionais (tapes) induz alterações positivas no padrão de marcha e no controlo postural, nomeadamente no aumento da amplitude média de dorsi-flexão da tíbio-társica aquando do ataque do pé ao solo, contribuindo para a redução da postura em equino-varus, bem como proporciona uma actividade muscular mais graduada entre os músculos que rodeiam a articulação tíbiotársica, tendo-se verificado um aumento da contracção do tibial-anterior associado a uma inibição da actividade do tricípete sural. Contudo, mesmo após três meses de uso continuado das ligaduras funcionais ainda se verificam diferenças entre os grupos de controlo e experimental, em algumas variáveis, tanto cinéticas como cinemáticas ou electromiográficas, incluindo a área de migração do centro de pressão, o que nos sugere que o tempo determinado para a utilização dos tapes poderá não ter sido suficiente para a obtenção de uma normalização do padrão de marcha e para a aquisição de um controlo postural mais efectivo. Será pertinente salientar ainda que, poderá ser necessário o uso de mais sistemas de contenção, para além dos tapes, para se conseguir obter um padrão de marcha dito normal.

\section{CORRESPONDÊNCIA \\ Leandro Machado}

Laboratório de Biomecânica

Faculdade de Desporto

Universidade do Porto

Rua Dr. Plácido Costa, 91

4200-450 Porto

Portugal

email: Imachado@ fade.up.pt 


\section{REFERÊNCIAS BIBLIOGRÁFICAS}

1. Abel MF, Juhl GA, Vaughan CL, Damiano DL (1998). Gait assessment of fixed ankle-foot orthoses in children with spastic diplegia. Arch Phys M ed Rehabil 79, 126-132.

2. Barros ALP, Silva AM, Oliveira LF, Imbirida LA, Garcia MAC (1999). Índice Estabilométrico em suporte Uni/Bipodal. Biomecânica da Postura e do Movimento. VII Congresso Brasileiro de Biomecânica 3: 241-244.

3. Bartlett DJ, Palisano RJ (2000). A multivariate model of determinants of motor change for children with cerebral palsy. Physical Therapy 80, 598-612.

4. Bobath B (1967). The very early treatment of cerebral palsy. Developmental M edicine and Child Neurology 9: 373-390.

5. Celso CF, Muniz RA, Oliveira LF, Imbirida LA, Garcia MC (2001). Limites de estabilidade antero-posterior de adultos normais. Biomecânica da postura e do movimento. IX Congresso Brasileiro de Biomecânica 2:148-151.

6. Condie DN, Bader DL, Pratt DJ (1993). Biomechanical basis of orthotic management. Oxford: Heinemann.

7. Cordova ML, Ingersoll CD, Palmieri RM (2002). Efficacy of prophylatic ankle support: an experimental perspective. Journal of A thletic Training 37(4), 446-457.

8. Correia PP, Santos PM, Veloso A (1993). Electromiografia Fundamentação fisiológica, M étodos de recolha e Processamento, A plicações cinesiológicas. $1^{\text {a }}$ ed. Lisboa: Edições FMH.

9. Cusick BD (1988). Managing foot deformity in children with neuromotor disorders. Physical Therapy 68: 1903-1912.

10. De Leva P (1995). Joint center longitudinal positions computed from a selected subset of chandler's data. Journal of Biomechanics 29(9): 1231-1233.

11. DeLuca PA (1992). The child's foot and ankle. New York: Raven Press, Ltd.

12. Dimeglio A, Bonnet F (1997). Reeducation du pied bot varus equin. Kinesithérapie - M edecine physique - Readaptation $26: 428-438$.

13. Duarte M, Zatsiorsky VM (2002). Effects of body lean and visual information on the equilibrium maintenance during stance. Exp Brain Research 146: 60-69.

14. Hagberg B, Olow I (1996). The changing panorama of cerebral palsy in Sweden. Prevalence and origin during the birth year period 1987-90. A cta Pediatrica 85: 954-960.

15. Horta L. (1993). Ligaduras funcionais na actividade desportiva. Revista Portuguesa de M edicina Desportiva 11: 125-136.

16. Knackfuss IG, Rosenbum S, Gomes ES (1993). Análise Biomecânica do Pé- Comportamento das Pressões na Região Plantar. V Congresso Brasileiro de Biomecânica, 29-31.
17. Macdonald R. (1990). Taping Thechniques- Principles and Practice. London: Butterworth Heinemann.

18. Machado S. (2005). O efeito da palmilha proprioceptiva na marcha de crianças com hemiplegia espástica por paralisia cerebral. Revista Portuguesa de Ciências do D esporto (artigo submetido).

19. Mueller K, Cornwall M, McPoil T, Mueller D (1995). Effect of two contemporary tone inhibiting ankle foot orthoses on foot-loading patterns in adult hemiplegics: a small group study. Top Stroke R ehabilitation 1(4): 1-16.

20. Murphy B (1999). The use of adhesive ankle strapping and bracing in the prevention of ankle injury and the effect on the proprioception properties of the athlete. Journal of Sports M edicine 23(3): 147-149.

21. Neville B, Goodman R (2000). Congenital Hemiplegia. London: Mac Keith Press.

22. Nurse MA, Nigg BM, Stefanyshyn DJ, Liu W, Miller JE (1998). Differences in the sensation of the plantar surface of the human foot. North A merican Congress on Biomechanics, 14-18.

23. Olney SJ, Griffin MP, Monga TN, McBride JD (1991). Work and power in gait of stroke patients. A rchives of M edical Rehabilitation 72: 309-314.

24. O’Sullivan SB, Schmitz J (1993). Fisioterapia- Avaliação e Tratamento. $2^{a}$ ed. São Paulo: Editora Manole.

25. Perez V, Greve P, Yoshizumi L, Morini J (2001). Effect of the bandage kinesio taping in spasticity in cerebral palsy of dipareticcase report. Department of Physical Therapy of University Mogi das Cruzes.

26. Perry SD, McIlroy WE, Norrie RG, Maki BE (1998). Mechanical Facilitation of Sensation from plantar footsurface boundaries: effects on postural stabilization. N orth A merican Congress on Biomechanics, 14-18.

27. Shieppati M, Hugon M, Grasso M, Nardone A, Galante M (1994). The limits of equilibrium in young and elderly normal subjects in parkinsonians. Electroencephalography and Clinical Neurophysiology 93: 286-295.

28. Sussman MD (1991). The Diplegic Child. Rosemont: American Academy of Orthopaedic Surgeons.

29. Thomas SS, Mazur JM, Wright N (1992). Quantitative assessment of ankle foot orthoses for children with cerebral palsy. Development M edical Child Neurology 34: 547-555.

30. Winter DA (1990). Biomechanics and motor control of human movement. $2^{\text {nd }}$ ed. New York: John Wiley \&Sons.

\footnotetext{
${ }^{1}$ Referir-nos-emos frequentemente às ligaduras funcionais pela forma inglesa - Tapes - de uso comum em Portugal.
} 